\title{
Immune-checkpoint inhibitor plus chemotherapy versus conventional chemotherapy for first-line treatment in advanced non-small cell lung carcinoma: a systematic review and meta-analysis
}

Yixin Zhou ${ }^{1,2,3 \dagger}$, Chen Chen ${ }^{1,2,4 \dagger}$, Xuanye Zhang ${ }^{1,2,5 \dagger}$, Sha Fu ${ }^{6 \dagger}$, Cong Xue $e^{1,2,5 \dagger}$, Yuxiang Ma ${ }^{1,2,5}$, Wenfeng Fang ${ }^{1,2,5}$, Yunpeng Yang ${ }^{1,2,5}$, Xue Hou ${ }^{1,2,5}$, Yan Huang ${ }^{1,2,5}$, Hongyun Zhao ${ }^{1,2,5}$, Shaodong Hong ${ }^{1,2,5^{*}}$ (D) and Li Zhang ${ }^{1,2,5^{*}}$

\begin{abstract}
Background: Immune-checkpoint inhibitors plus chemotherapy are emerging as effective first-line treatment in advanced non-small-cell lung carcinoma (NSCLC), but little is known about the magnitude of benefits and potential clinical predictors.

Methods: We performed a meta-analysis of randomized trials that compared PD-1/PD-L1 inhibitor plus chemotherapy with chemotherapy in first line of treatment for advanced NSCLC. The outcomes included progression-free survival (PFS), overall survival (OS), objective response rate (ORR) and treatment-related adverse events (AEs). A fixed-effect or random-effects model was adopted depending on between-study heterogeneity.

Results: Six trials involving 3144 patients were included. PD-1/PD-L1 inhibitor plus chemotherapy was significantly associated with improvement of PFS (hazards ratio [HR], 0.62; 95\% Cl 0.57-0.67; $P<.001)$, OS (HR, 0.68; 95\% Cl 0.53-0.87; $P=.002$ ) and ORR (relative ratio $[R R], 1.56 ; 95 \%$ Cl 1.29-1.89; $P<.001$ ), irrespective of PD-L1 expression level. The significant predictor(s) for treatment benefit with combination therapy versus chemotherapy alone were PD-L1 expression level for PFS $(P<.001)$; types of checkpoint inhibitor for ORR $(P<.001)$; histology $(P=.025)$, age $(P=.038)$, gender $(P<.001)$, and types of checkpoint inhibitor $(P<.001)$ for OS. In safety analyses, PD-1/ PD-L1 inhibitor plus chemotherapy had significantly higher incidence of adverse events (AEs) of grade 3 or higher (RR, 1.14; $P=.007)$, AEs leading to treatment discontinuation (RR, 1.29; $P=.022)$, serious AEs (RR 1.70; $P=.006)$, immune mediated AEs of any grade (RR, 2.37; $P<.001)$, and immune mediated AEs of grade 3 or higher (RR, 3.71; $P<.001)$.
\end{abstract}

Conclusions: PD-1/PD-L1 inhibitor plus chemotherapy, compared with chemotherapy, is associated with significantly improved PFS, ORR, and OS in first-line therapy in NSCLC, at the expense of increased treatment-related AES.

Keywords: Immune checkpoint inhibitor, Programmed death 1, Programmed death 1 ligand 1, Chemotherapy, Non-small cell lung carcinoma, Predict

\footnotetext{
* Correspondence: hongshd@sysucc.org.cn; zhangli6@mail.sysu.edu.cn

${ }^{\dagger}$ Yixin Zhou, Chen Chen, Xuanye Zhang, Sha Fu and Cong Xue contributed equally to this work.

${ }^{1}$ State Key Laboratory of Oncology in South China, Guangzhou, China

Full list of author information is available at the end of the article
}

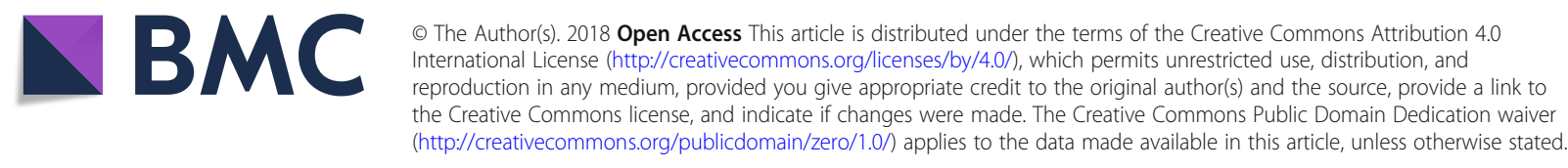




\section{Background}

Advanced non-small-cell lung cancer (NSCLC) remains the leading cause of cancer-related mortality worldwide $[1,2]$. Platinum-based chemotherapy has been the standard of care for the first-line treatment of advanced NSCLC that lacks targetable driver mutations [3]. However, chemotherapy is associated with only modest efficacy and has reached a plateau. With recent advance of immune checkpoint inhibitors treatment that block the PD-L1 (programmed cell death 1 ligand 1) and PD-1 (programmed cell death 1) pathway, pembrolizumab monotherapy has replaced chemotherapy as the first-line treatment for patients with PD-L1 tumor proportion score (TPS) of at least 50\% [4], and pembrolizumab plus platinum and pemetrexed for those with nonsquamous histology irrespective of PD-L1 expression $[5,6]$.

Preclinical evidence suggested that chemotherapeutic agents may exert immune-potentiating effects under certain condition [1], exemplified by increasing the mutational load in cancer cells which leads to a higher chance of neoantigen presentation [7], augmenting major histocompatibility complex class I and human leucocyte antigen (HLA)-A, B, C expression [8, 9], reducing the activity of immune-suppressive cells [10,11], and increasing the sensitivity of tumor cells to T-cell effector cytokines [12]. Chemotherapy has also been shown to induce PD-L1 expression on tumor cells $[13,14]$. Thus, combining immunotherapy and chemotherapy might synergistically improve the antitumor activity of anti-PD-1 and anti-PDL1 monotherapy, which have been demonstrated in several randomized controlled trials $[5,6,15]$.

Despite the promising activity of immuno-oncology (IO) combinatorial treatment, there remain several unanswered questions. For example, will the IO-chemotherapy combinatorial regimens lead to improved efficacy in all comers at the expense of increased toxicity? Are there any clinical or molecular factors that could predict benefit of IO-chemotherapy combination?

Because the magnitude of IO-chemotherapy benefits remains controversial and individual trials were not powerful enough to explore a difference of treatment effect between patient subgroups, a meta-analysis of currently available trials comparing PD-1/PD-L1 inhibitor plus chemotherapy with chemotherapy will provide important and clinically useful information.

\section{Method}

\section{Study eligibility and identification}

Eligible randomized controlled trials that compared PD-1/PD-L1 inhibitor plus chemotherapy with chemotherapy in the first-line setting were identified from Pubmed, Embase and the Cochrane Central Register of Controlled Trials. We searched for studies that published in English from inception to June 10, 2018, using the keywords including pembrolizumab, nivolumab, atezolizumab, durvalumab, PD-1, PD-L1, non-small cell lung cancer, and randomized controlled trial (Additional file 1: Supplementary Method). We also reviewed abstracts from major conference proceedings of the American Society of Clinical Oncology (ASCO), the European Society of Medical Oncology (ESMO), the American Association for Cancer Research (AACR), and the World Conference on Lung Cancer (WCLC). When duplicate studies were identified, only the most complete and updated data of the study was included.

\section{Data extraction}

Two authors (Y.X.Z. and C.C.) independently extracted data with a predefined information sheet. Discrepancies were resolved by consensus. We extracted the following items for each included trial: acronym and design of the trial, number of patients enrolled, year of publication or conference presentation, clinicopathological characteristics of the patients including PD-L1 level, type of chemotherapeutic agents and IO drug, and treatment outcomes including progression-free survival (PFS), overall survival (OS), objective response rate (ORR), duration of response, and treatment-related adverse events (AEs). When we needed additional information that were not provided, we contacted the corresponding authors to request it. Two independent reviewers (S.D.H. and X.Y.Z.) conducted the risk of bias assessment of the included trials with the Cochrane Collaboration's tool [16].

\section{Statistical analysis}

The primary objective was to investigate the association between IO-chemotherapy vs. chemotherapy and treatment effects (PFS, OS, and ORR) in patients with advanced NSCLC. The PFS and OS outcomes were measured with hazards ratios (HRs) and the corresponding 95\% confidence intervals (CIs) which were extracted from each study or calculated using other available statistics. The secondary outcome was the pooled risk of adverse events.

We used the Cochrane's Q statistic to assess between-study heterogeneity, and calculated the $I^{2}$ statistic, which estimates the percentage of total variation across studies due to heterogeneity rather than chance. The pooled estimates for PFS and OS were presented with HRs, 95\% CIs and $P$ values calculated using the inverse-variance-weighted method, while the measures for dichotomous data (ORR and frequency of adverse events) were pooled with the risk ratios (RRs), 95\% CIs and $P$ values using the Mantel Haenszel method. The random effect models were chosen if obvious heterogeneity was present $\left(I^{2}>50 \%\right)$, otherwise the fixed effect models were applied [16]. 
To investigate the sources of heterogeneity, predefined subgroup analyses were performed. Tests of interaction were used to assess the differences in treatment effect across these subgroups. Publication bias was evaluated by examining the funnel plot of the effect size for each trial against the reciprocal of its SE, together with the Egger test. Sensitivity analyses of treatment efficacy were also conducted by: 1) repeating the analyses by omitting one study at a time; 2) repeating the analysis by removing studies that were only available from conference presentation; and 3) using both fixed-effects and random-effects models for the analysis. We used Stata version 15.0 (Stata Corporation, College Station, TX) for all of the analyses. The nominal level of significance was set at $5 \%$.

\section{Results}

A total of 1329 studies were identified through the initial search strategy. After screening the abstracts and reviewing the full texts, a total of six trials $[5,6,15,17-20]$ involving 3144 patients were included in the final analyses (Additional file 2: Figure S1). The assessment of risk of bias was provided in Additional file 3: Table S1. All the trials were well designed and reported. The main source of bias was that data in three trials (CheckMate 227, KEYNOTE-407, and Impower131) could only be retrieved from conference presentations [17-19].

The main characteristics of the included trials were summarized in Table 1 and Additional file 3: Table S2. The patient characteristics were well balanced between the experimental and control groups in all trials. Three trials $[5,6,15,20]$ enrolled patients with nonsquamous NSCLC, two trials $[17,19]$ with squamous NSCLC, and one trial [18] with both squamous and nonsquamous patients. Four trials $[5,6,18-20]$ investigated anti-PD-1 antibody and two trials $[15,17]$ investigated anti-PD-L1 antibody. All six trials used standard-of-care chemotherapeutic regimens as recommended by practice guidelines.

The main outcomes of the included trials were summarized in Additional file 3: Table S3. The median follow-up time ranged from 7.8 to 23.9 months. All six trials provided PFS, ORR and DOR data; OS data was not reported in CheckMate 227 study.

\section{Benefit of IO-chemotherapy combination}

The pooled result showed that IO-chemotherapy combination significantly reduced the risk of disease progression compared with chemotherapy (HR, 0.62; 95\% CI $0.57-0.67 ; \mathrm{z}=11.06, P<.001$ ) (Additional file 2: Figure $\mathrm{S} 1 \mathrm{~A})$. There was no significant heterogeneity in the overall treatment effect in terms of PFS across the six trials $\left(I^{2}=42.3 \%, X^{2}=8.66, P=.123\right)$. The funnel plot for the PFS revealed no asymmetry (Additional file 2: Figure S2A; Egger test $P=.713$ ), indicating no obvious publication bias regarding PFS.

In terms of OS benefit, the IO-chemotherapy combination led to a $32 \%$ reduction in the risk of death compared with chemotherapy (HR, 0.68; 95\% CI 0.53-0.87; $\mathrm{z}=3.04$, $P=.002$ ) (Fig. 1b). The objective response rate was also significantly improved with the IO-chemotherapy combination (pooled RR, 1.56; 95\% CI 1.29 to $1.89 ; \mathrm{z}=4.52, P$ $<.001$ ) (Fig. 1c). Significant heterogeneity was observed in the analysis of OS $\left(I^{2}=77.3 \%, X^{2}=17.61, P=.001\right)$ and ORR $\left(I^{2}=77.6 \%, X^{2}=22.36, P<.001\right)$, respectively. The funnel plots for the OS and ORR revealed no asymmetry (Additional file 2: Figures $\mathrm{S} 2 \mathrm{~B}$ and $\mathrm{C}$; Egger

Table 1 Characteristics of Patients Comparing IO-Chemotherapy with Chemotherapy in Included Trials

\begin{tabular}{|c|c|c|c|c|c|c|c|c|c|c|c|}
\hline \multirow[t]{2}{*}{ Source } & \multirow[t]{2}{*}{ PD-(L)1 Drug } & \multirow[t]{2}{*}{ Histology } & \multicolumn{2}{|c|}{ No. of patients ${ }^{a}$} & \multirow[t]{2}{*}{$\begin{array}{l}\text { Median age } \\
\text { (years) }^{a}\end{array}$} & \multirow[t]{2}{*}{$\begin{array}{l}\text { Male } \\
(\%)^{a}\end{array}$} & \multicolumn{2}{|c|}{$\begin{array}{l}\text { Performance } \\
\text { status }^{a}\end{array}$} & \multicolumn{3}{|c|}{ PD-L1 subgroups ${ }^{a}$} \\
\hline & & & $\mathrm{ITT}$ & As treated & & & $\begin{array}{l}\text { ECOG } 0 \\
(\%)\end{array}$ & $\begin{array}{l}\text { ECOG } 1 \\
(\%)\end{array}$ & $<1 \%(\%)$ & $\begin{array}{l}1-49 \% \\
(\%)\end{array}$ & $\begin{array}{l}\geq 50 \% \\
(\%)\end{array}$ \\
\hline $\begin{array}{l}\text { KEYNOTE-189 } \\
2018 \text { [6] }\end{array}$ & Pembrolizumab & nonsquamous & 410 vs 206 & 405 vs 202 & 65 vs 64 & 62 vs 53 & 45 vs 39 & 54 vs 61 & 31 vs 31 & 31 vs 28 & 32 vs 34 \\
\hline $\begin{array}{l}\text { IMpower150 } \\
2018 \text { [15] }\end{array}$ & Atezolizumab & nonsquamous & 400 vs 400 & 393 vs 394 & 63 vs 63 & 60 vs 60 & 39 vs 43 & 60 vs 57 & 47 vs 50 & 33 vs 31 & 20 vs 19 \\
\hline $\begin{array}{l}\text { KEYNOTE-021 } \\
2016 \text { [5], } 2018 \\
{[20]}\end{array}$ & Pembrolizumab & nonsquamous & 60 vs 63 & 59 vs 62 & 63 vs 63 & 37 vs 41 & 40 vs 46 & 58 vs 54 & 35 vs 37 & 32 vs 37 & 33 vs 27 \\
\hline $\begin{array}{l}\text { KEYNOTE-407 } \\
2018\end{array}$ & Pembrolizumab & squamous & 278 vs 281 & 278 vs 280 & 65 vs 65 & 79 vs 84 & 26 vs 32 & 74 vs 68 & 34 vs 35 & 37 vs 37 & 26 vs 26 \\
\hline $\begin{array}{l}\text { IMpower131 } \\
2018 \text { [17] }\end{array}$ & Atezolizumab & squamous & 343 vs 340 & 334 vs 334 & 65 vs 65 & 81 vs 82 & 34 vs 32 & 66 vs 68 & 47 vs 50 & 38 vs 36 & 15 vs 14 \\
\hline $\begin{array}{l}\text { CheckMate } \\
2272018 \text { [18] }\end{array}$ & Nivolumab & $\begin{array}{l}\text { suqamous and } \\
\text { nonsquamous }\end{array}$ & 177 vs 186 & 172 vs 185 & 64 vs 64 & 73 vs 67 & 33 vs 31 & 66 vs 68 & 100 vs 100 & 0 vs 0 & 0 vs 0 \\
\hline
\end{tabular}

"Data presented as "IO-chemotherapy group vs chemotherapy group"

bembrolizumab (200 mg, Q3W), Atezolizumab (1200 mg, Q3W), Nivolumab (360 mg, Q3W)

Abbreviation: 10 immuno-oncology, ITT intention-to-treat 
A Progression-Free Survival

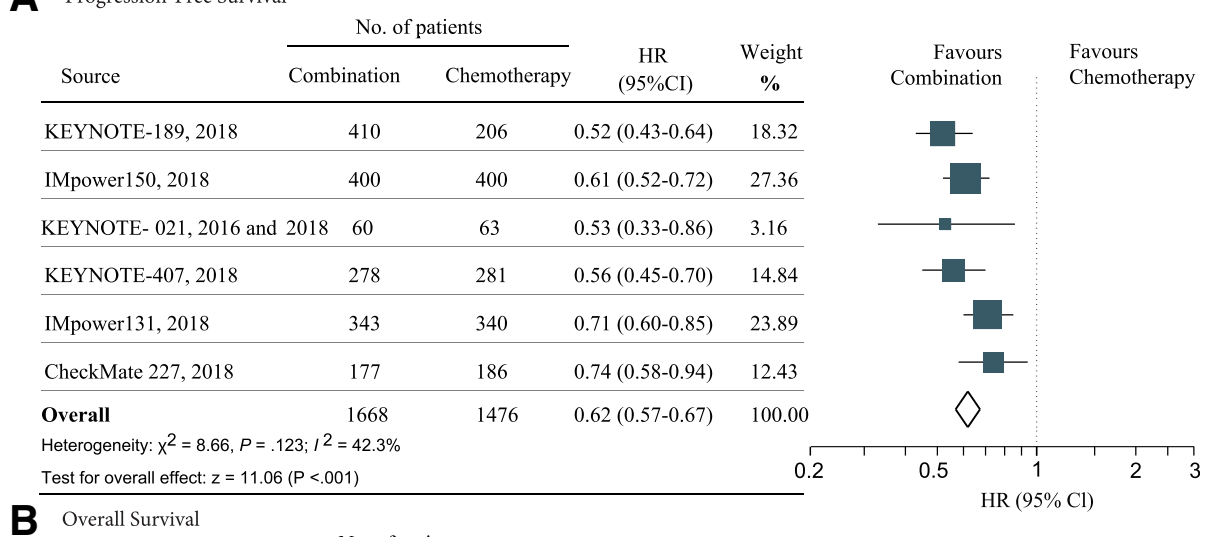

B Overall Survival

No. of patients

\begin{tabular}{|c|c|c|c|c|}
\hline \multirow[b]{2}{*}{ Source } & & \multirow{2}{*}{$\begin{array}{c}\text { HR } \\
(95 \% \mathrm{CI}) \\
\end{array}$} & \multirow{2}{*}{$\begin{array}{c}\text { Weigl } \\
\%\end{array}$} \\
\hline & Combination & Chemotherapy & & \\
\hline KEYNOTE-189, 2018 & 410 & 206 & $0.49(0.38-0.64)$ & \\
\hline IMpower150, 2018 & 400 & 400 & $0.76(0.63-0.93)$ & \\
\hline KEYNOTE-021, 2016 and 20 & $018 \quad 60$ & 63 & $0.56(0.32-0.95)$ & \\
\hline KEYNOTE-407, 2018 & 278 & 281 & $0.64(0.49-0.85)$ & \\
\hline IMpower131, 2018 & 343 & 340 & $0.96(0.78-1.18)$ & \\
\hline Overall & 1491 & 1290 & $0.68(0.53-0.87)$ & 100 \\
\hline \multicolumn{5}{|c|}{ Heterogeneity: $x^{2}=17.61, P=.001 ; \prime^{2}=77.3 \%$} \\
\hline \multicolumn{4}{|c|}{ Test for overall effect: $z=3.04(P=.002)$} & \\
\hline
\end{tabular}

Test for overall effect: $z=3.04(P=.002)$

\section{2}

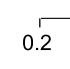

C Objective Response Rate

\begin{tabular}{|c|c|c|c|c|c|c|c|c|}
\hline \multirow{3}{*}{ Source } & \multicolumn{4}{|c|}{ No. of patients } & \multicolumn{2}{|c|}{$\begin{array}{r}\text { Favours } \\
\text { Chemotherapy }\end{array}$} & \multirow[t]{3}{*}{$\begin{array}{l}\text { Favours } \\
\text { Combination }\end{array}$} & \\
\hline & \multicolumn{2}{|c|}{ Combination } & \multicolumn{2}{|c|}{ Chemotherapy } & \multirow{2}{*}{$\begin{array}{c}\mathrm{RR} \\
(95 \% \mathrm{CI})\end{array}$} & \multirow{2}{*}{$\begin{array}{c}\text { Weight } \\
\%\end{array}$} & & \\
\hline & Events & Total & Events & Total & & & & \\
\hline KEYNOTE-189, 2018 & 195 & 410 & 39 & 206 & $2.51(1.86-3.39)$ & 14.92 & & \\
\hline IMpower150, 2018 & 226 & 356 & 161 & 336 & $1.32(1.15-1.51)$ & 20.98 & & \\
\hline KEYNOTE- 021, 2016 and 2018 & 34 & 60 & 19 & 63 & $1.88(1.21-2.91)$ & 10.63 & & \\
\hline KEYNOTE-407, 2018 & 161 & 278 & 108 & 281 & $1.51(1.26-1.80)$ & 19.50 & & \\
\hline IMpower131, 2018 & 169 & 343 & 140 & 340 & $1.20(1.01-1.41)$ & 19.95 & & \\
\hline CheckMate 227, 2018 & 65 & 177 & 43 & 186 & $1.59(1.15-2.20)$ & 14.02 & & \\
\hline Overall & 850 & 1668 & 1476 & 1668 & $1.56(1.29-1.89)$ & 100.00 & & \\
\hline \multicolumn{5}{|c|}{ Heterogeneity: $x^{2}=22.36, P<.001 ; 1^{2}=77.6 \%$} & & & & \\
\hline \multirow{2}{*}{\multicolumn{5}{|c|}{ Test for overall effect: $z=4.52(P<.001)$}} & 0.3 & 0.5 & 2 & 3 \\
\hline & & & & & \multicolumn{3}{|c|}{$\mathrm{RR}(95 \% \mathrm{Cl})$} & \\
\hline
\end{tabular}

Fig. 1 Forest plot of hazard ratios and risk ratios comparing (a) progression-free survival, (b) overall survival, and (c) objective response rate in patients who received IO-Chemotherapy vs Chemotherapy alone. Squares represent study-specific effect size (HR or RR). The area of square is inversely proportional to the standard error of the study (and therefore indirectly to the sample size) and larger area indicates greater weight in the calculation of the pooled effect size. The horizontal line crossing the square represents the $95 \% \mathrm{Cl}$. The diamonds represent the estimated overall effect, based on the meta-analysis. $\mathrm{HR}$, hazard ratio; $\mathrm{RR}$, relative risk; Cl, confidence interval

test: $P=.370$ for $\mathrm{OS}$ analysis and $P=.308$ for ORR analysis, respectively).

\section{Subgroup analyses}

The number of trials with available data for subgroupanalysis was summarized in Additional file 3: Table S4. Results of the subgroup-analyses for PFS, OS and ORR were summarized in Fig. 2, Additional file 2: Figures S3 and S4.

\section{Subgroup analyses by PD-L1 expression level}

PD-1/PD-L1 inhibitor plus chemotherapy led to statistically longer PFS across all tested subgroups of PD-L1 expression level, including those with a PD-L1 TPS of less than $1 \%(\mathrm{HR}, 0.76 ; 95 \% \mathrm{CI}, 0.67-0.86 ; P<.001$; 


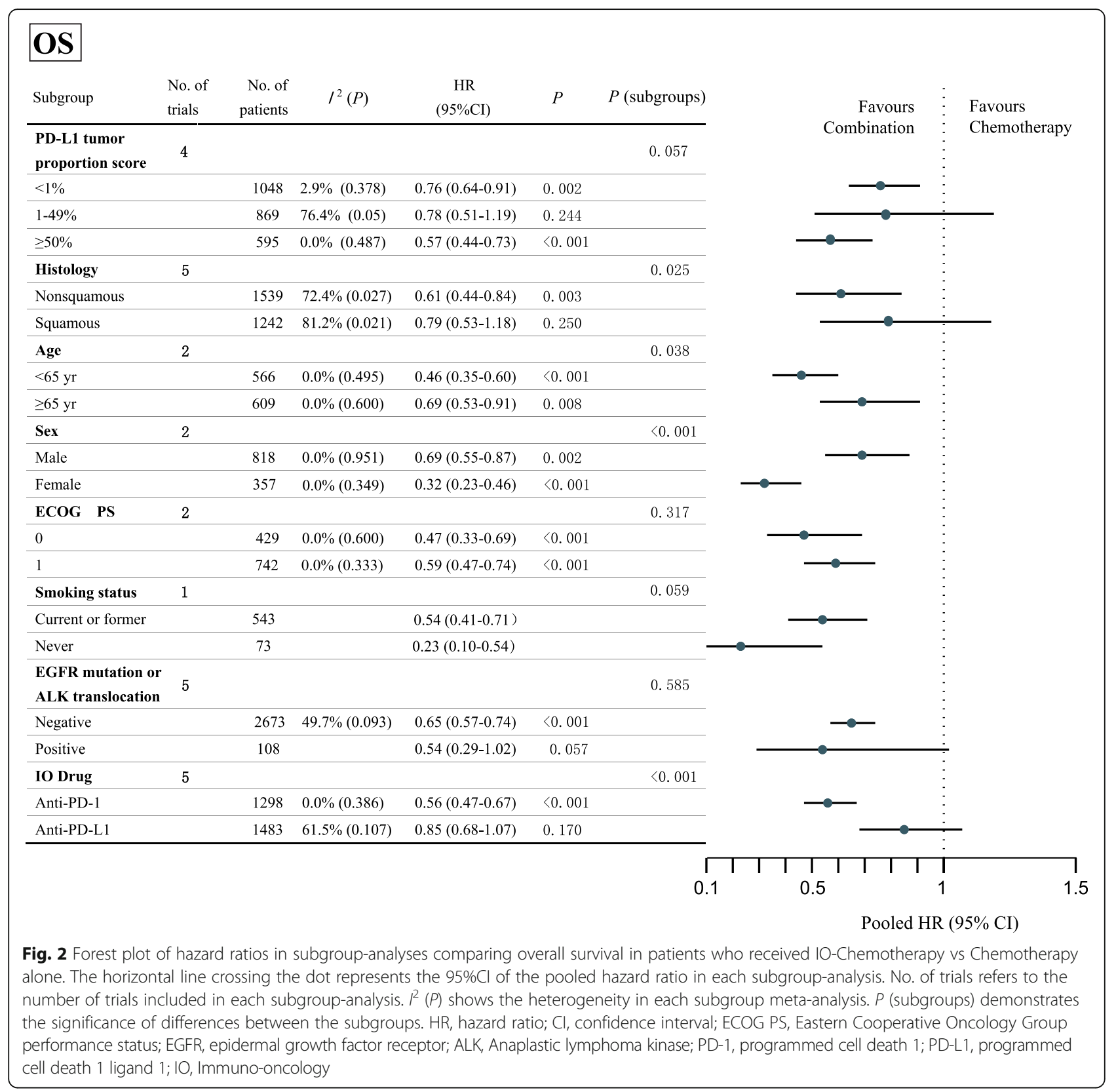

heterogeneity, $P=.952$ ), a score of 1 to $49 \%$ (HR, 0.60; 95\% CI, 0.51-0.71; $P<.001$; heterogeneity, $P=.635)$, and a score of at least $50 \%(\mathrm{HR}, 0.38 ; 95 \% \mathrm{CI}, 0.31-0.47 ; P$ <.001; heterogeneity, $P=.928$ ) (Fig. 3). The magnitude of PFS benefit was significantly different among subgroups of PD-L1 TPS $(P<.001)$.

For patients in whom the PD-L1 TPS was less than $1 \%$, the pooled HR for OS was 0.76 (95\% CI, 0.640.91; $P=.002$; heterogeneity, $P=.378$ ), compared with the HR of 0.78 (95\% CI, $0.51-1.19 ; P=.244$; heterogeneity, $P=.050)$ in those with a score of 1 to $49 \%$ and 0.57 (95\% CI, 0.44-0.73, $P<.001$; heterogeneity, $P$ $=.487$ ) in those with a score of $50 \%$ or greater
(Additional file 2: Figure S3). The difference of OS benefit across PD-L1 subgroups obtained a near-significant trend $(P=.057)$.

The response rate was the highest in patients with a PD-L1 TPS of at least 50\% (RR, 1.95; 95\% CI 1.34-2.82; $P<.001$; heterogeneity, $P=.093)$. In the subgroup with a score between 1 and $49 \%$, the pooled RR was 1.39 (95\% CI 0.98-1.96; $P=.062$; heterogeneity, $P=.079$ ). In the subgroup with a score of less than $1 \%$, the pooled RR was 1.54 (95\% CI 1.16-2.05; $P=.003$; heterogeneity, $P=.064)$. There was no significant interaction between treatment effect in terms of ORR and PD-L1 expression level $(P=.232)$. 


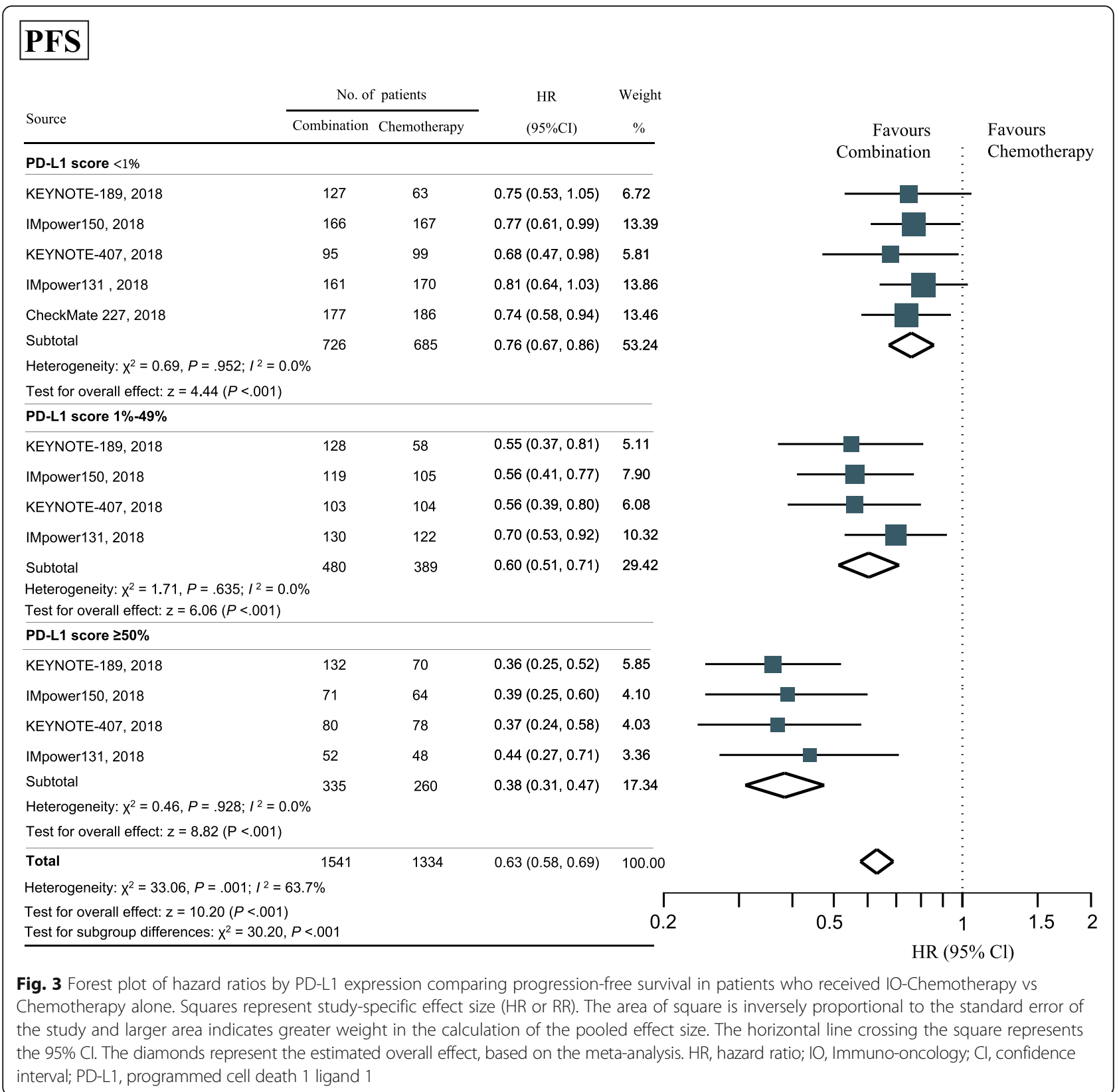

\section{Subgroup analyses by other factors}

None of the other factors predicted PFS benefit with the IO-chemotherapy combination vs. chemotherapy (Additional file 2: Figure S3), including histology (nonsquamous HR, 0.59 vs Squamous HR,0.65; interaction, $P=.217)$, age $(<65$ years $H R, 0.60$ vs $\geq 65$ years HR, 0.67; interaction, $P=.377$ ), sex (male HR, 0.65 vs Female HR, 0.60; interaction, $P=.365$ ), ECOG performance status (PS $=0 \mathrm{HR}, 0.61$ vs $\mathrm{PS}=1 \mathrm{HR}, 0.64$; interaction, $P=.629$ ), smoking status (current or former HR, 0.61 vs Never HR, 0.68; interaction, $P$ $=.525$ ), genomic alterations in EGFR or ALK (negative HR, 0.62 vs positive HR, 0.59 ; interaction, $P=.860$ ), and type of IO drug (anti-PD-1 HR, 0.58 vs anti-PD-L1, 0.65; interaction, $P=.179$ ).

However, there were several other factors that could predict OS and ORR benefit from the IO-chemotherapy over chemotherapy alone (Fig. 2 and Additional file 2: Figure S4). Patients with nonsquamous histology (nonsquamous $\mathrm{HR}, 0.61$ vs squamous HR, 0.79 ; interaction, $P=.025)$, younger age $(<65$ years $H R, 0.46$ vs $\geq 65$ years $\mathrm{HR}, 0.69$; interaction, $P=.038$ ), who were females (female HR, 0.32 vs male HR, 0.69; interaction, $P<.001$ ), and who received anti-PD-1 antibody (anti-PD-1 antibody HR, 0.56 vs anti-PD-L1 antibody HR, 0.85; interaction, $P<.001)$ might receive more OS benefit from the 
combination therapy. And patients with anti-PD-1 drug might have a higher objective response rate from the combination therapy than those with anti-PD-L1 antibody (anti-PD-1 RR, 1.81 vs anti-PD-L1 RR 1.27; interaction, $P<.001)$.

\section{Sensitivity analyses}

To evaluate the robustness of the combined outcomes, we carried out sensitivity analyses by omitting specific studies or altering statistical models. The results showed that the overall estimates remained consistent across these analyses (Additional file 2: Figure S5; Additional file 3: Tables S5 and S6).

\section{Safety analyses}

Safety analyses were conducted in patients who had received at least one dose of the study drug (Table 1). The median or mean duration of treatment is summarized in Additional file 3: Table S3, and the number of patients included for each safety analysis is presented in Fig. 4. The pooled results demonstrated that IO-chemotherapy combination was significantly associated with higher frequency of treatment-related AEs of grade 3 or more severity (pooled RR 1.14, 95\% CI 1.04-1.26, $P=.007$ ), AEs leading to treatment discontinuation (pooled RR 1.29, 95\% CI 1.01-1.60, $P=.022$ ), treatment-related serious AEs (pooled RR 1.70, 95\% CI 1.17-2.49, $P=.006$ ), immune mediated AEs of any grade (pooled RR 2.37, 95\% CI $1.98-2.84, P<.001)$, and immune mediated AEs of grade 3 or more severity (pooled RR $3.71,95 \%$ CI $2.63-$
5.24, $P<.001)$. However, there was no significant difference in the frequency of AEs of any grade (pooled RR $1.03,95 \%$ CI $0.99-1.06, P=.132$ ), death attributed to treatment (pooled RR 1.14, 95\% CI 0.80-1.63, $P=.47$ ) and immune mediated AEs leading to death (pooled RR 2.24, 95\% CI 0.42-11.8, $P=.343$ ).

\section{Discussion}

To the best of our knowledge, this is the first meta-analysis that focuses on investigating the association between PD-1/PD-L1 inhibitor plus chemotherapy versus chemotherapy and treatment outcomes in patients with advanced NSCLC. The analysis demonstrates that PD-1/PD-L1 inhibitor plus chemotherapy is statistically associated with a $38 \%$ reduction in the risk of disease progression, a $32 \%$ reduction in the risk of death, and 1.6 times the probability of achieving an objective response compared with standard chemotherapy for first-line treatment of advanced NSCLC, though at the expense of increased treatment-related adverse events. The significant predictor(s) for treatment benefit with IO-chemotherapy combination vs chemotherapy were PD-L1 expression for PFS; IO drug type for ORR; histology, age, gender, and IO drug type for OS.

One plausible point about IO combination therapy is its potential for increasing the percentage responders who otherwise do not respond to monotherapy. In the KEYNOTE-024 and -042 studies, pembrolizumab leads to statistically significant and clinically meaningful improvement of OS in patients with PD-L1 TPS of 50\% or

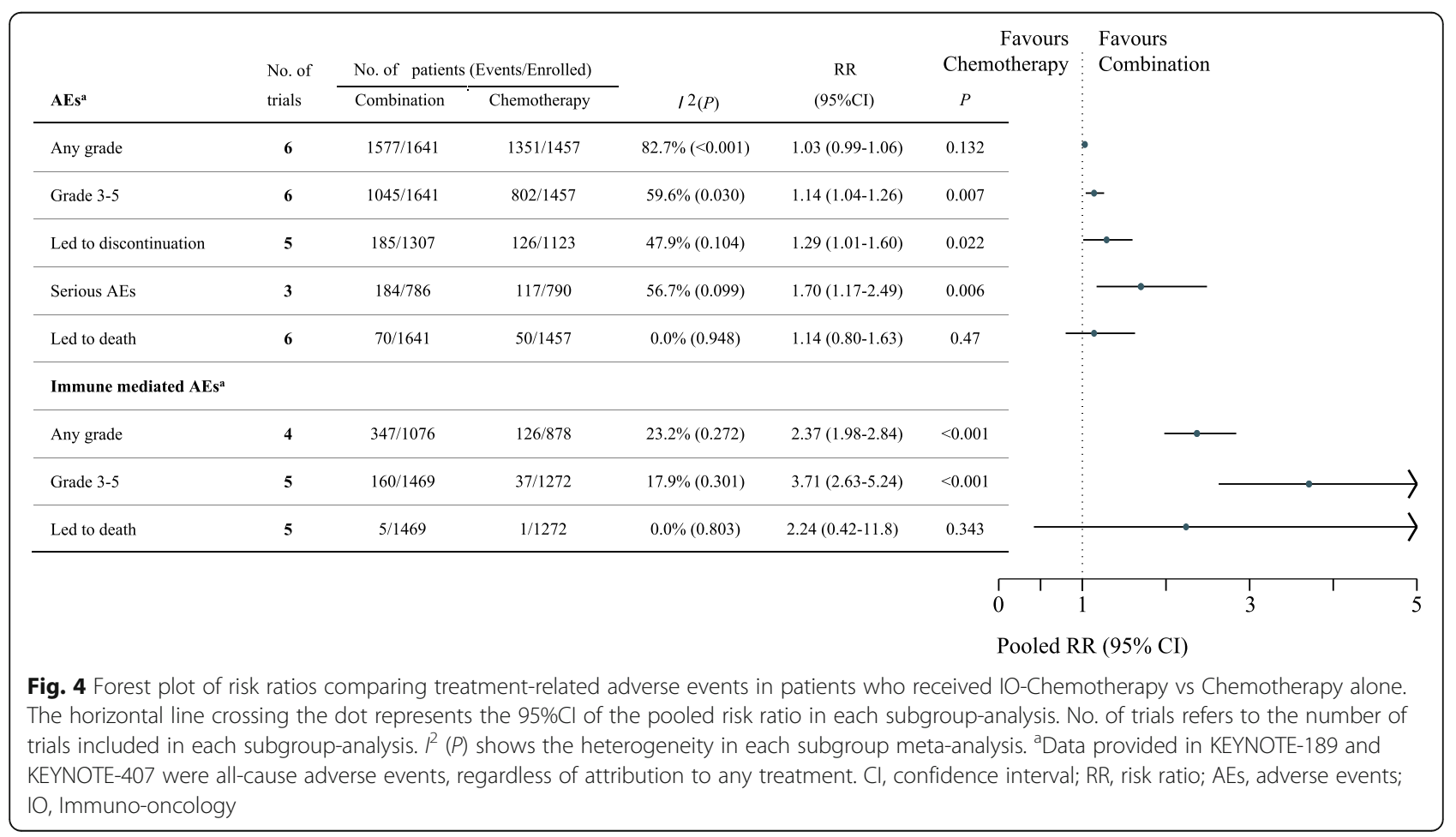


greater (HR, 0.63 and 0.69 , respectively) $[4,21]$; however there is no significant OS difference in patients with PD-L1 TPS of $1-49 \%$ (HR, 0.92). This is clinically relevant because patients with high PD-L1 expression represent a minority of those with NSCLC [22]. And less than one half of patients with advanced NSCLC ever receive second-line therapy due to rapid deterioration during disease progression $[22,23]$. Therefore, maximizing the chance of response to first-line treatment is important. In the present study, we found that PD-1/PD-L1 inhibitor plus chemotherapy led to improved survival across all tested subgroups of patients according to PD-L1 expression, including patients with low or negative PD-L1 expression. These findings in unselected patients are particularly relevant because the majority of patients have tumors with low, negative, or undetectable PD-L1 expression [22]. Furthermore, there are multiple challenges regarding using PD-L1 as a predictive marker of immunotherapy, including the intratumoral heterogeneity and interassay discordance of PD-L1 expression [24-26]. Nevertheless, the greatest benefit was observed in the subgroup with a PD-L1 TPS of $50 \%$ or more (HR for OS, 0.57). This finding also raises a clinically important question whether PD-1/PD-L1 inhibitor plus chemotherapy has greater efficacy than the single agent PD-1/ PD-L1 inhibitor in these patients, which needs to be directly compared in randomized studies.

In subgroup analysis, there was a significant difference in treatment benefits between PD-1 inhibitor and PD-L1 inhibitor when combined with chemotherapy (HR for OS, 0.56 vs 0.85 ; interaction, $P<.001$; RR for ORR, 1.81 vs 1.27 ; interaction, $P<.001)$. Similarly, a previous network meta-analysis demonstrates that the probability of treatment ranking was higher with PD-1 inhibitor than PD-L1 inhibitor for previously treated NSCLC [27]. One possible explanation is that anti-PD-L1 antibody spares the interaction between PD-1 and PD-L2 [28]. However, cautions should be exercised in deciding which drug class is preferred due to the lack of head-to-head clinical trials.

Our study also reveals a statistically significant interaction between histology and OS benefit (nonsquamous HR, 0.61 vs squamous HR, 0.79 ; interaction $P=.025$ ). This seems consistent with the previous belief that patients with squamous NSCLC receive less benefit from checkpoint inhibitors [29]. Nevertheless, this subgroup analysis does not deny the benefits of IO-chemotherapy for squamous NSCLC. Rather, exploratory biomarker analysis and extended follow-up are needed to fully evaluate the role of IO-chemotherapy in patients with squamous NSCLC, in whom treatment options have been very limited for decades and the prognosis remains poor.

Interestingly, a recent meta-analysis demonstrates that the relative benefit of immunotherapy (predominantly single agent) is greater in male cancer patients than in female patients [30]. In contrast with this report, our study demonstrates greater OS benefit with PD-1/PD-L1 inhibitor plus chemotherapy for female patients than for male patients (female HR, 0.32 vs male HR, 0.69; interaction $P$ $<.001)$. However, it should be noted that the subgroup analysis of OS regarding gender was only available from the pembrolizumab studies, KEYNOTE-189 [6] and KEYNOTE-407 [19]. Therefore, it remains controversy whether the magnitude of OS benefit with immune checkpoint inhibitors are sex-dependent. Finally, whether the relatively lower efficacy of immunotherapy in female patients could be reversed by applying combinatorial treatment strategy as evident in this analysis warrants further investigation.

We also showed that IO-chemotherapy significantly improved OS both in patients < 65 years old (HR 0.46; $P$ $<.001)$ and $\geq 65$ years old (HR 0.69; $P=.008$ ), with greater benefit in in younger patients (interaction $P=.038$ ). This finding is different from previous meta-analyses which revealed similar efficacy with immune checkpoint inhibitor monotherapy in patients younger vs. older than 65 years old [31,32]. These results are clinically relevant because a majority of lung cancer patients were diagnosed at older ages and were often with poor performance status in real world [33]. Considering the higher frequency of treatment-related adverse events with IO-chemotherapy, it remains an open question how to tailor the treatment for older patients to optimize clinical outcomes in the era of immunotherapy.

This meta-analysis further unfolded that smoking history, EGFR mutation or $A L K$ rearrangement, and PS 0 or 1 were not predictive of OS benefit with IO-chemotherapy vs chemotherapy. Typically, patients with EGFR or ALK genomic alterations receive little OS advantage with the single agent PD-1/PD-L1 inhibitor [34]. Despite the high PD-L1 expression in oncogene-addicted tumors [35, 36], they are associated with a high frequency of inactive tumor-infiltrating lymphocytes [37], low mutation load [38], and weak immunogenicity [39]. These factors are hypothesized to account for the inferior efficacy of immunotherapy in patients with EGFR- or ALK-driven NSCLC. However, the IMpower 150 study showed that combination therapy with atezolizumab, bevacizumab and chemotherapy significantly improved survival in these patients [15]. It remains unclear whether it is the addition of chemotherapy or anti-angiogenesis agent or both that reverse the "cold" immune microenvironment in oncogene-driven NSCLC.

Regarding the safety profiles, the addition of PD-1/ PD-L1 inhibitor to chemotherapy was significantly associated with increased risk of developing AEs of grade 3 or worse severity, treatment-related drug discontinuation and serious AEs. However, the frequency of deaths attributed to treatment was similar between the both 
groups. Immune-mediated adverse events were more frequently observed in the IO-chemotherapy group, with frequency and severity consistent with those noted in the PD-1/PD-L1 inhibitor monotherapy [40].

A strength of this work is the quality of evidence available and used in the meta-analysis. Source data were obtained from six randomised controlled trials that involved over 3000 patients. Thus, the meta-analysis could overcome the problem of inadequate power of each individual trial by pooling data together. Albeit the strength above, we encountered several limitations during this study. First of all, our meta-analysis relies on published results rather than on individual patients' data. Therefore, the results from subgroup analysis remain inconclusive but merely suggestive. The optimal clinical and molecular predictors of benefit from IO-chemotherapy remains to be elucidated. Secondly, the clinical trials included in this meta-analysis enrolled highly selective patients, e.g. patients with good performance status, sufficient organ functions and no comorbidities like autoimmune disease. Therefore, we were unable to explore the effect of these regimens in patients who were ineligible for clinical trials. And these patients represent a large proportion in real-world clinical practice. Finally, the OS data from the included trials were not mature enough. An update meta-analysis with final OS data will be important in the future. Yet, this meta-analysis established the definite benefit of IO-chemotherapy vs chemotherapy in terms of PFS and ORR in the first line setting, which might serve as moderate surrogate endpoints for OS [41].

In conclusion, PD-1/PD-L1 inhibitor plus chemotherapy, compared with chemotherapy, significantly prolonged PFS and OS in first-line of treatment for advanced NSCLC, irrespective of PD-L1 expression level. Future studies are needed to explore reliable predictors of treatment efficacy and to determine which chemotherapeutic modality will improve patient's survival in combination with PD-1/PD-L1 inhibitor. Finally, the trade-off between benefits and risk of side effects as well as treatment costs should be considered in clinical practice.

\section{Additional files}

\section{Additional file 1: Supplementary Method. Search strategies for}

PubMed, EMBASE, and Cochrane database. (PDF $314 \mathrm{~kb}$ )

Additional file 2 Figure S1. Trial selection process. Figure S2. Funnel plot comparing hazard ratios for (A) progression-free survival and (B) overall survival, and risk ratios for (C) objective response rate. Each study's effect estimate plotted against its standard error. The outer dashed lines represent the confidence interval boundary within which $95 \%$ of studies are expected to lie in the absence of bias or heterogeneity. The solid vertical line represents the summary treatment effect. Figure S3. Forest plot of hazard ratios in subgroup-analyses comparing progression-free survival in patients who received IO-Chemotherapy vs Chemotherapy alone. The horizontal line crossing the dot represents the $95 \% \mathrm{Cl}$ of the pooled hazard ratio in each subgroup-analysis. No. of trials refers to the number of trials included in each subgroup-analysis. $P^{2}(P)$ shows the heterogeneity in each subgroup meta-analysis. $P$ (subgroups) demonstrates the significance of differences between the subgroups. $\mathrm{Cl}$, confidence interval; ECOG PS, ECOG performance-status score; and IO, Immunooncology. Figure S4. Forest plot of risk ratios in subgroup-analyses comparing objective response rate in patients who received IOChemotherapy vs Chemotherapy alone. The horizontal line crossing the dot represents the $95 \% \mathrm{Cl}$ of the pooled risk ratio in each subgroup-analysis. No. of trials refers to the number of trials included in each subgroup-analysis. I $^{2}$ $(P)$ shows the heterogeneity in each subgroup meta-analysis. $P$ (subgroups) demonstrates the significance of differences between the subgroups. IO, Immuno-oncology. Figure S5. Sensitivity analyses of progression-free survival (PFS), overall survival (OS), objective response rate (ORR) by repeating the pooled analyses with one study omitted at a time. (PDF $609 \mathrm{~kb}$ )

Additional file $\mathbf{3}$ Table S1. Quality assessment: risk of bias by Cochrane Collaboration's tool. Table S2. Additional characteristics of patients comparing IO-Chemotherapy with Chemotherapy in Included trials. Table S3. Main outcomes of the included trials. Table S4. Summary of the data status for subgroup-analyses among the included trials. Table S5. Summary of sensitivity analyses results using both fixed-effects and random-effects models. Table $\mathbf{S 6}$. Summary of sensitivity analyses after removing studies that were only available from conference presentation. (PDF $982 \mathrm{~kb})$

\section{Abbreviations}

AACR: American Association for Cancer Research; AEs: Adverse events; ASCO: American Society of Clinical Oncology; Cl: Confidence interval; ECOG PS: ECOG performance-status score; ESMO: European Society of Medical Oncology; HLA: Human leucocyte antigen; HR: Hazard ratio; IO: Immunooncology; NSCLC: Non-small-cell lung carcinoma; ORR: Objective response rate; OS: Overall survival; PD-1: Programmed cell death 1; PD-

L1: Programmed cell death 1 ligand 1; PFS: Progression-free survival; RR: Risk ratio; TPS: Tumor proportion score tumor proportion score; WCLC: World Conference on Lung Cancer

\section{Acknowledgments}

We sincerely appreciate Yidu Cloud Corporation (Beijing, China) for data management and Mrs. Yan Lei (Medical Science Liaison, Bristol-Myers Squibb, Beijing, China) for retrieval of source data. We also thank Dr. Yaxiong Zhang, Zhonghan Zhang, Shen Zhao, Huaqiang Zhou, Gang Chen, Xi Chen, Fangfang Gao, Yuanyuan Zhao, and Ningning Zhou (Sun Yat-sen University Cancer Center) for their help in reviewing the manuscript.

\section{Funding}

This study was funded by grants 2016YFC0905500 and 2016YFC0905503 from the National Key R\&D Program of China; 81602005, 81702283, and 81602011 from the National Natural Science Funds of China; 2017B020227001 from the Science and Technology Program of Guangdong Province. The funding sources had no role in the design and conduct of the study; collection, management, analysis, and interpretation of the data; preparation, review, or approval of the manuscript; and decision to submit the manuscript for publication.

Availability of data and materials

All data generated or analysed during this study are included in the published article.

\section{Authors' contributions}

$Y Z, C C$ and XZ contributed to data acquisition, data interpretation, and statistical analysis and drafting of the manuscript. SF and CX contributed to data acquisition, data interpretation, and statistical analysis. SH and LZ contributed to the study design, data acquisition, data interpretation, statistical analysis. All the other authors (YM, WF, YY, XH, YH, and $H Z)$ contributed to data acquisition and critical revision of the manuscript. All the authors have final approval of the submitted manuscript and reached agreement to be accountable for all aspects of the work.

Ethics approval and consent to participate Not applicable. 


\section{Consent for publication}

Not applicable.

\section{Competing interests}

Li Zhang has received research support from AstraZeneca, Eli Lilly and company, and Roche. No other disclosures were reported.

\section{Publisher's Note}

Springer Nature remains neutral with regard to jurisdictional claims in published maps and institutional affiliations.

\section{Author details}

${ }^{1}$ State Key Laboratory of Oncology in South China, Guangzhou, China. ${ }^{2}$ Collaborative Innovation Center for Cancer Medicine, Guangzhou, China. ${ }^{3}$ Department of VIP region, Sun Yat-sen University Cancer Center, Guangzhou, China. ${ }^{4}$ Department of Radiotherapy, Sun Yat-sen University Cancer Center, Guangzhou, China. ${ }^{5}$ Department of Medical Oncology, Sun Yat-sen University Cancer Center, 651 Dongfeng East Road, Guangzhou 510060, China. ${ }^{6}$ Guangdong Provincial Key Laboratory of Malignant Tumor Epigenetics and Gene Regulation, Pathology Department, Sun Yat-Sen Memorial Hospital, Sun Yat-Sen University, Guangzhou, China.

\section{Received: 15 August 2018 Accepted: 6 December 2018} Published online: 22 December 2018

\section{References}

1. Apetoh L, Ladoire S, Coukos G, Ghiringhelli F. Combining immunotherapy and anticancer agents: the right path to achieve cancer cure? Ann Oncol. 2015;26:1813-23.

2. Global Burden of Disease Cancer C, Fitzmaurice C, Akinyemiju TF, Al Lami FH, Alam T, Alizadeh-Navaei R, et al. Global, regional, and national cancer incidence, mortality, years of life lost, years lived with disability, and disability-adjusted life-years for 29 cancer groups, 1990 to 2016: a systematic analysis for the global burden of disease study. JAMA Oncol. 2018;4(11): 1553-1568.

3. Hanna N, Johnson D, Temin S, Baker S Jr, Brahmer J, Ellis PM, et al. Systemic therapy for stage IV non-small-cell lung cancer: American Society of Clinical Oncology clinical practice guideline update. J Clin Oncol. 2017;35:3484-515.

4. Reck M, Rodriguez-Abreu D, Robinson AG, Hui R, Csoszi T, Fulop A, et al. Pembrolizumab versus chemotherapy for PD-L1-positive non-small-cell lung cancer. N Engl J Med. 2016;375:1823-33.

5. Langer CJ, Gadgeel SM, Borghaei H, Papadimitrakopoulou VA, Patnaik A, Powell SF, et al. Carboplatin and pemetrexed with or without pembrolizumab for advanced, non-squamous non-small-cell lung cancer: a randomised, phase 2 cohort of the open-label KEYNOTE-021 study. Lancet Oncol. 2016;17:1497-508.

6. Gandhi L, Rodriguez-Abreu D, Gadgeel S, Esteban E, Felip E, De Angelis F, et al. Pembrolizumab plus chemotherapy in metastatic non-small-cell lung cancer. N Engl J Med. 2018;378:2078-92.

7. Szikriszt B, Poti A, Pipek O, Krzystanek M, Kanu N, Molnar J, et al. A comprehensive survey of the mutagenic impact of common cancer cytotoxics. Genome Biol. 2016;17:99.

8. de Biasi AR, Villena-Vargas J, Adusumilli PS. Cisplatin-induced antitumor immunomodulation: a review of preclinical and clinical evidence. Clin Cancer Res. 2014;20:5384-91.

9. Gravett AM, Trautwein N, Stevanovic S, Dalgleish AG, Copier J. Gemcitabine alters the proteasome composition and immunopeptidome of tumour cells. Oncoimmunology. 2018;7:e1438107.

10. Suzuki E, Kapoor V, Jassar AS, Kaiser LR, Albelda SM. Gemcitabine selectively eliminates splenic gr-1+/CD11b+ myeloid suppressor cells in tumor-bearing animals and enhances antitumor immune activity. Clin Cancer Res. 2005;11: 6713-21.

11. Kodumudi KN, Woan K, Gilvary DL, Sahakian E, Wei S, Djeu JY. A novel chemoimmunomodulating property of docetaxel: suppression of myeloidderived suppressor cells in tumor bearers. Clin Cancer Res. 2010;16:4583-94.

12. Hato SV, Khong A, de Vries IJ, Lesterhuis WJ. Molecular pathways: the immunogenic effects of platinum-based chemotherapeutics. Clin Cancer Res. 2014;20:2831-7.

13. Song Z, Yu $X$, Zhang $Y$. Altered expression of programmed death-ligand 1 after neo-adjuvant chemotherapy in patients with lung squamous cell carcinoma. Lung Cancer. 2016;99:166-71.
14. Peng J, Hamanishi J, Matsumura N, Abiko K, Murat K, Baba T, et al. Chemotherapy induces programmed cell death-ligand 1 overexpression via the nuclear factor-kappaB to Foster an immunosuppressive tumor microenvironment in ovarian Cancer. Cancer Res. 2015;75:5034-45.

15. Socinski MA, Jotte RM, Cappuzzo F, Orlandi F, Stroyakovskiy D, Nogami N, et al. Atezolizumab for first-line treatment of metastatic nonsquamous NSCLC. N Engl J Med. 2018;378:2288-301.

16. Higgins JPGS, editor. Cochrane collaboration. Cochrane handbook for systematic reviews of Interventions. West Sussex: Wiley; 2008.

17. Jotte RM, Cappuzzo F, Vynnychenko I, Stroyakovskiy D, Abreu DR, Hussein MA, et al. IMpower131: primary PFS and safety analysis of a randomized phase III study of atezolizumab + carboplatin + paclitaxel or nab-paclitaxel vs carboplatin + nab-paclitaxel as $1 \mathrm{~L}$ therapy in advanced squamous NSCLC. J Clin Oncol. 2018;36:9000. https://doi.org/10.1200/JCO.2018.36.18_ suppl.LBA9000.

18. Borghaei H, Hellmann MD, Paz-Ares LG, Ramalingam SS, Reck M, O'Byrne KJ, et al. Nivolumab (Nivo) + platinum-doublet chemotherapy (chemo) vs chemo as first-line (1L) treatment (Tx) for advanced non-small cell lung cancer (NSCLC) with < 1\% tumor PD-L1 expression: results from CheckMate 227. J Clin Oncol. 2018;36:9001. https://doi.org/10.1200/JCO.2018.36.15_ suppl.9001.

19. Paz-Ares LG, Luft A, Tafreshi A, Gumus M, Mazieres J, Hermes B, et al. Phase 3 study of carboplatin-paclitaxel/nab-paclitaxel (chemo) with or without pembrolizumab (Pembro) for patients (pts) with metastatic squamous (Sq) non-small cell lung cancer (NSCLC). J Clin Oncol. 2018;36:105. https://doi. org/10.1200/JCO.2018.36.15_suppl.105.

20. Gentzler RD, Langer CJ, Borghaei H, Gadgeel SM, Papadimitrakopoulou V, Patnaik A, et al. 24-month overall survival from KEYNOTE-021 cohort G: Pemetrexed-carboplatin plus pembrolizumab as first-line therapy for advanced nonsquamous NSCLC. J Clin Oncol. 2018;36:9026. https://doi.org/ 10.1200/JCO.2018.36.15_suppl.9026.

21. Lopes G, Wu Y-L, Kudaba I, Kowalski D, Cho BC, Castro G, et al. Pembrolizumab (pembro) versus platinum-based chemotherapy (chemo) as first-line therapy for advanced/metastatic NSCLC with a PD-L1 tumor proportion score (TPS) $\geq 1 \%$ : open-label, phase 3 KEYNOTE-042 study. J Clin Oncol. 2018;36:LBA4. https://doi.org/10.1200/JCO.2018.36.18_suppl.LBA4.

22. Rimm DL, Han G, Taube JM, Yi ES, Bridge JA, Flieder DB, et al. A prospective, multi-institutional, pathologist-based assessment of 4 immunohistochemistry assays for PD-L1 expression in non-small cell lung cancer. JAMA Oncol. 2017;3:1051-8.

23. Davies J, Patel M, Gridelli C, de Marinis F, Waterkamp D, McCusker ME. Realworld treatment patterns for patients receiving second-line and third-line treatment for advanced non-small cell lung cancer: a systematic review of recently published studies. PLoS One. 2017;12:e0175679.

24. Pinato DJ, Shiner RJ, White SD, Black JR, Trivedi P, Stebbing J, et al. Intratumoral heterogeneity in the expression of programmed-death (PD) ligands in isogeneic primary and metastatic lung cancer: implications for immunotherapy. Oncoimmunology. 2016:5:e1213934.

25. Munari E, Zamboni G, Lunardi G, Marchionni L, Marconi M, Sommaggio M, et al. PD-L1 expression heterogeneity in non-small cell lung cancer: defining criteria for harmonization between biopsy specimens and whole sections. J Thorac Oncol. 2018;13(8):1113-1120.

26. Hui R, Garon EB, Goldman JW, Leighl NB, Hellmann MD, Patnaik A, et al. Pembrolizumab as first-line therapy for patients with PD-L1-positive advanced non-small cell lung cancer: a phase 1 trial. Ann Oncol. 2017;28: 874-81.

27. You W, Liu M, Miao JD, Liao YQ, Song YB, Cai DK, et al. A network metaanalysis comparing the efficacy and safety of anti-PD-1 with anti-PD-L1 in non-small cell lung cancer. J Cancer. 2018;9:1200-6.

28. Zou W, Wolchok JD, Chen L. PD-L1 (B7-H1) and PD-1 pathway blockade for cancer therapy: mechanisms, response biomarkers, and combinations. Sci Transl Med. 2016;8:328rv4.

29. Gettinger S, Rizvi NA, Chow LQ, Borghaei H, Brahmer J, Ready N, et al. Nivolumab monotherapy for first-line treatment of advanced non-small-cell lung cancer. J Clin Oncol. 2016;34:2980-7.

30. Conforti F, Pala L, Bagnardi V, De Pas T, Martinetti M, Viale G, et al. Cancer immunotherapy efficacy and patients' sex: a systematic review and metaanalysis. Lancet Oncol. 2018;19:737-46.

31. Wu Y, Ju Q, Qian B, Zhang F, Shi H. The effectiveness of PD-1 inhibitors in non-small cell lung cancer (NSCLC) patients of different ages. Oncotarget. 2018;9:7942-8. 
32. Elias R, Giobbie-Hurder A, McCleary NJ, Ott P, Hodi FS, Rahma O. Efficacy of PD-1 \& PD-L1 inhibitors in older adults: a meta-analysis. J Immunother Cancer. 2018;6:26.

33. Moro-Sibilot D, Smit E, de Castro Carpeno J, Lesniewski-Kmak K, Aerts J, Villatoro $R$, et al. Outcomes and resource use of non-small cell lung cancer (NSCLC) patients treated with first-line platinum-based chemotherapy across Europe: FRAME prospective observational study. Lung Cancer. 2015;88:215-22.

34. Lee CK, Man J, Lord S, Cooper W, Links M, Gebski V, et al. Clinical and molecular characteristics associated with survival among patients treated with checkpoint inhibitors for advanced non-small cell lung carcinoma: a systematic review and meta-analysis. JAMA Oncol. 2018;4:210-6.

35. Chen N, Fang W, Zhan J, Hong S, Tang Y, Kang S, et al. Upregulation of PDL1 by EGFR activation mediates the immune escape in EGFR-driven NSCLC: implication for optional immune targeted therapy for NSCLC patients with EGFR mutation. J Thorac Oncol. 2015:10:910-23.

36. Hong S, Chen N, Fang W, Zhan J, Liu Q, Kang S, et al. Upregulation of PD-L1 by EML4-ALK fusion protein mediates the immune escape in ALK positive NSCLC: implication for optional anti-PD-1/PD-L1 immune therapy for ALKTKIs sensitive and resistant NSCLC patients. Oncoimmunology. 2016;5: e1094598.

37. Martin P, Spitzmueller A, Wu S, Widmaier M, KORN R, Althammer S, et al. Mutually exclusive expression of CD73 and PDL1 in tumors from patients (pt) with NSCLC, gastroesophageal (GE) and urothelial bladder carcinoma (UBC). J Clin Oncol. 2017;35:3079.

38. Rizvi NA, Hellmann MD, Snyder A, Kvistborg P, Makarov V, Havel JJ, et al. Cancer immunology. Mutational landscape determines sensitivity to PD-1 blockade in non-small cell lung cancer. Science. 2015;348:124-8.

39. Dong ZY, Zhang JT, Liu SY, Su J, Zhang C, Xie Z, et al. EGFR mutation correlates with uninflamed phenotype and weak immunogenicity, causing impaired response to PD-1 blockade in non-small cell lung cancer. Oncoimmunology. 2017;6:e1356145.

40. Pillai RN, Behera M, Owonikoko TK, Kamphorst AO, Pakkala S, Belani CP, et al. Comparison of the toxicity profile of PD-1 versus PD-L1 inhibitors in nonsmall cell lung cancer: a systematic analysis of the literature. Cancer. 2018; 124:271-7.

41. Nakashima K, Horita N, Nagai K, Manabe S, Murakami S, Ota E, et al. Progression-free survival, response rate, and disease control rate as predictors of overall survival in phase III randomized controlled trials evaluating the first-line chemotherapy for advanced, locally advanced, and recurrent non-small cell lung carcinoma. J Thorac Oncol. 2016;11:1574-85.

Ready to submit your research? Choose BMC and benefit from:

- fast, convenient online submission

- thorough peer review by experienced researchers in your field

- rapid publication on acceptance

- support for research data, including large and complex data types

- gold Open Access which fosters wider collaboration and increased citations

- maximum visibility for your research: over $100 \mathrm{M}$ website views per year

At BMC, research is always in progress.

Learn more biomedcentral.com/submissions 